\title{
Radiation swelling and hardness of high-entropy alloys based on the Ti-Ta-Nb-V system irradiated with krypton ions
}

I.A. Ivanov ${ }^{1,2}$, A. Ryskulov ${ }^{1}$, A. Kurakhmedov ${ }^{1}$, A. Kozlovskiy ${ }^{1,2^{*}}$, D. Shlimas ${ }^{1,2}$, M.V. Zdorovets $^{1,2,3}$, V.V. Uglov ${ }^{4}$, S.V. Zlotski ${ }^{4}$, J. Ke $^{5}$

${ }^{1}$ The Institute of Nuclear Physics of Republic of Kazakhstan, Almaty, Kazakhstan

${ }^{2}$ L.N. Gumilyov Eurasian National University, Nur-Sultan, Kazakhstan

${ }^{3}$ Ural Federal University, Yekaterinburg, Russia

${ }^{4}$ Belarusian State University, Minsk, Belarus

${ }^{5}$ Beijing Institute of Technology Beijing, China

*E-mail: kozlovskiy.a@inp.kz

\begin{abstract}
The aim of this work is to study the effect of irradiation with low-energy $\mathrm{Kr}^{14+}$ ions with an energy of $280 \mathrm{keV}$ on radiation swelling and a decrease in the strength characteristics of highentropy alloys based on the Ti-Ta-Nb-V system. The prospects of these studies are due to the possibility of obtaining new data on the radiation resistance of high-entropy alloys, which have great potential for use as structural materials for new generation reactors. The choice of this type of irradiation and a dose of $5 \times 10^{15} \mathrm{Kr} / \mathrm{cm}^{2}$ made it possible to simulate the effect of radiation swelling arising at a displacement per atom of 30-50 dpa in a small surface layer no more than 100-150 nm thick. During the studies carried out, it was found that the formed medium- and high-entropy alloys $\mathrm{TaNbV}$ and $\mathrm{TiTaNbV}$ have increased resistance to swelling and deformation of the crystal structure. At the same time, alloys of the $\mathrm{NbV}$ and $\mathrm{TaNbV}$ type have the highest degree of resistance to softening, while the TiTaNbV alloy has a lower resistance to swelling, since the addition of titanium leads to a decrease in the density and hardness of the alloy.
\end{abstract}

Keywords: high-entropy alloys, substitution solid solution, radiation damage, swelling, radiation resistance, hardness

\section{Introduction}

One of the promising areas of research in modern materials science is the study of properties and methods of obtaining high-entropy alloys based on refractory elements [1-3]. The interest in these types of alloys, as well as the methods for their preparation, is due to significant differences in mechanical and strength characteristics compared to traditional alloys and metals. At the same time, researchers are of particular interest to alloys based on refractory metals in combination with light $\mathrm{V}, \mathrm{Ti}, \mathrm{Cr}$ and heavy metals $\mathrm{Nb}$, Ta, W [4-8]. Such combinations make it possible to vary the density of alloys by adding light elements, while maintaining or increasing strength characteristics due to heavy refractory elements. As a rule, the structure of high-entropy alloys is characterized by substitutional solid solutions with a strongly deformed crystal lattice, the deformation of which is caused by differences in the atomic radii of the components $[9,10]$. The formation of such structures leads to an increase in the strength properties of alloys due to their high resistance to external influences. The most promising application of these alloys is nuclear power, in particular, the use as heat-resistant structural materials that will be used directly in the core of a nuclear reactor. According to a number of studies and earlier tests, these alloys can serve as an alternative to the currently used steels and ceramics [11-15].

However, in spite of all the above perspectives, there remains one important issue related to the radiation resistance of high-entropy alloys, as well as to the processes of radiation swelling at high radiation doses [16-18]. At the same time, it is of interest to study the radiation resistance and mechanisms of radiation damage not only when irradiated with heavy ions comparable in mass and energy with uranium fission fragments, but also the processes of radiation swelling and a decrease in the strength and mechanical properties of the near-surface layer of alloys at large values of atomic displacements induced radiation damage. As a rule, a thin near-surface layer 
with a thickness of up to several hundred nanometers is most susceptible to embrittlement during large-dose irradiation, and is also exposed to various external influences during operation. In the case of high irradiation doses, which are characterized by atomic displacements of 20-50 dpa, the processes of mixing and subsequent swelling and deformation are initiated in the structure of the surface layer $[19,20]$. According to the generally accepted theory, the structure of high-entropy alloys has a high resistance to deformation, while the more complex the alloy composition, the higher the degree of resistance to deformation and swelling [21-25]. It is also worth noting that the high melting points and thermal conductivity of high-entropy alloys make these materials one of the promising classes of candidate materials for nuclear power and rocket engineering.

Based on the above, the aim of this work is to study the resistance to swelling and deformation caused by irradiation with low-energy $\mathrm{Kr}^{14+}$ ions with an energy of $280 \mathrm{keV}$ and a dose of $5 \times 10^{15}$ $\mathrm{Kr} / \mathrm{cm}^{2}$. The choice of these irradiation conditions is associated with the possibility of modeling radiation damage processes at $\mathrm{dpa}=30-50$ (according to the results of SRIM Pro 2013 calculations) at a depth of 150-170 nm. High-entropy alloys based on the Ti-Ta-Nb-V system were selected as objects of research. As samples for comparison, a sample of pure vanadium and alloys $\mathrm{NbV}$, TaNbV, prepared by a similar technology for obtaining alloys, were selected. Radiation resistance was assessed using X-ray diffraction and Vickers hardness methods. The interest in these types of alloys, as well as in the study of their radiation resistance, is due to the interest in their practical application as structural materials due to their high heat resistance and refractory properties.

\section{Experimental part}

Samples of $\mathrm{V}, \mathrm{NbV}, \mathrm{TaNbV}$, TiTaNbV were manufactured at the Beijing Technological University by arc melting and casting in a copper cell, followed by vacuum annealing at a temperature of $1150^{\circ} \mathrm{C}$ for $24 \mathrm{~h}$, cold rolling to reduce the thickness by $85 \%$, and final annealing at a temperature of $1150^{\circ} \mathrm{C}$ for 72 hours.

The samples were irradiated at a DC-60 heavy ion accelerator located in Nur-Sultan, Kazakhstan. Low-energy $\mathrm{Kr}^{14+}$ ions with an energy of $280 \mathrm{keV}$ (20 keV/charge) were chosen as the type of ions. The irradiation fluence was $5 \times 10^{15} \mathrm{Kr} / \mathrm{cm}^{2}$. In order to avoid overheating, the targets during irradiation were placed on a water-cooled target holder. The calculation of energy losses was carried out in the SRIM Pro 2013 program code [26]. Figure 1 shows the results of modeling the displacements per atom and the concentration of implanted krypton in the samples under study. The maximum travel depth for krypton ions is $100-150 \mathrm{~nm}$, with the maximum damage at a depth of 60-70 $\mathrm{nm}$. According to the calculated data, the magnitude of the displacements at the maximum is from 30 to $50 \mathrm{dpa}$. The concentration of implanted krypton ions in the maximum damage was no less than 1 at. $\%$.

$280 \mathrm{keV}, 5^{*} 10^{15} \mathrm{~cm}^{-2}$

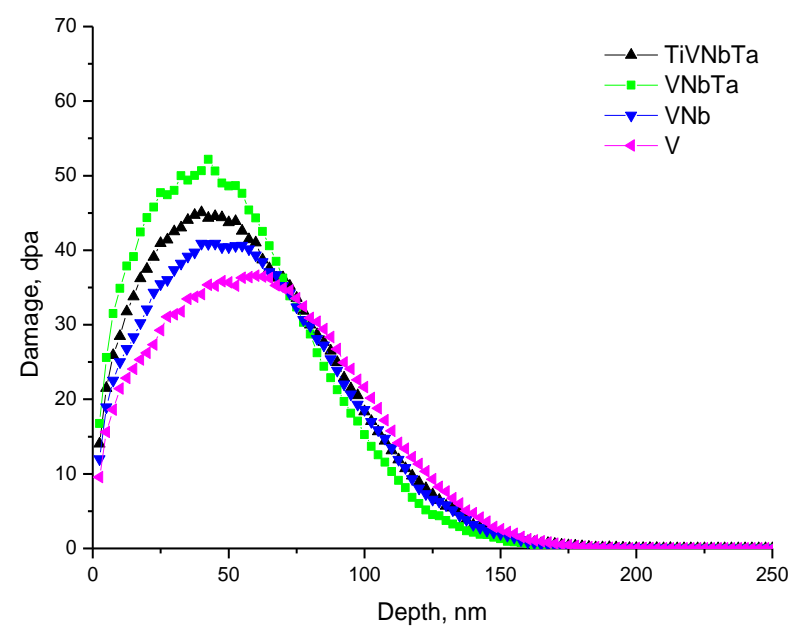

$280 \mathrm{keV}, 5^{*} 10^{15} \mathrm{~cm}^{-2}$

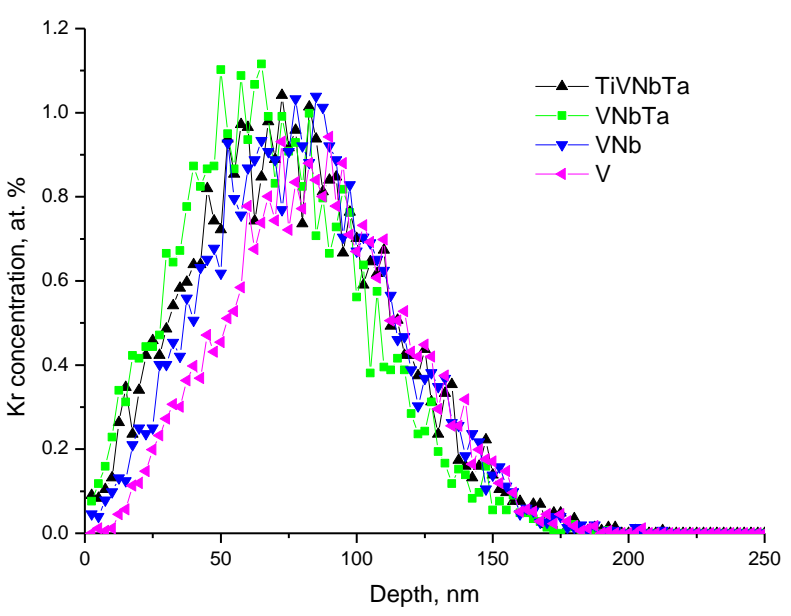


Figure 1. Profiles of radiation damage (a) and implanted krypton atoms (b) in depth for the samples under study, obtained using the SRIM Pro 2013 program code

The assessment of structural changes as a result of irradiation was carried out using the X-ray diffraction method, implemented using a D8 Advance ECO X-ray diffractometer. Bruker. Diffraction patterns were recorded in the Bragg-Brentano geometry, in the angular range $2 \theta=30$ $110^{\circ}$, with a step of $0.05^{\circ}$ using radiation with a wavelength of $1.54179 \AA$. Structural changes were determined using the DiffracEVA v.4.2 program code, which is based on a full-profile analysis method.

The phase composition was determined based on the analysis of the areas of diffraction peaks and their total contribution to the general diffraction pattern using the full-profile analysis method.

The irradiation effect on the change in structural properties was determined by the changes in interplanar distances, reflecting the deformation and distortion processes occurring in the structure. The deformation processes were changed along two main diffraction lines (110) and (200), reflecting the shift in the orientation of the texture.

The degree of swelling as a result of the radiation damage accumulation was determined based on the data of the change in the crystal lattice volume.

The strength properties and resistance of the investigated alloys to swelling and softening of the near-surface layer were determined using Vickers hardness test method. A diamond pyramid was used as an indenter; the pressure force on the indenter during testing was $1000 \mathrm{~N}$. An estimate of the difference in the change in hardness before and after irradiation of the samples under study made it possible to estimate the degree of softening and the degree of resistance of the surface layer to swelling. The softening value reflects the change in the hardness of the near-surface layer exposed to irradiation, as well as a decrease in damage and crack resistance.

\section{Results and discussion}

Figure 2 shows X-ray diffraction patterns before and after irradiation. The general view of the presented diffraction patterns (narrow and intense diffraction peaks) indicates a high crystallinity degree of the alloys, however, the shape of the diffraction lines (their asymmetry) is related to the possible both with the heterogeneity of the structure of the solid solution and for strongly deformed crystal lattices, which is characteristic of high-entropy alloys and is associated with the difference in the atomic radii of the selected elements.

For the sample of pure vanadium selected for comparison, in the initial state, the preferred orientation (200) is observed, as well as the body-centered type of the crystal lattice (PDF-00023-1058). After irradiation, a change in the preferred orientation to reorientation of grains along the (110) direction was revealed. At the same time, the shape of the diffraction lines after irradiation has a strongly pronounced asymmetry and a shift in the position of the diffraction line to the region of small angles is observed, which indicates the deformation of the vanadium lattice caused by irradiation.

The samples of $\mathrm{NbV}$ alloys are also characterized by the body-centered structure of the $\mathrm{NbV}$ substitutional solid solution phase (PDF-03-065-4353) with the $\operatorname{Im}-3 \mathrm{~m}(229)$ spatial system, with a preferred orientation (200). In this case, the asymmetric shape of reflections (211) and (310) indicates both the heterogeneity of the structure of the solid solution and a strong deformation of the structure in these directions associated with the substitutional solution. For irradiated samples of $\mathrm{NbV}$ alloys, there is no favorable orientation. In this case, both lines (211) and (310) are divided into two peaks. This is due to the formation of a deformed region upon irradiation with krypton ions. The diffraction line corresponding to this reflection is shifted to the region of smaller angles. 


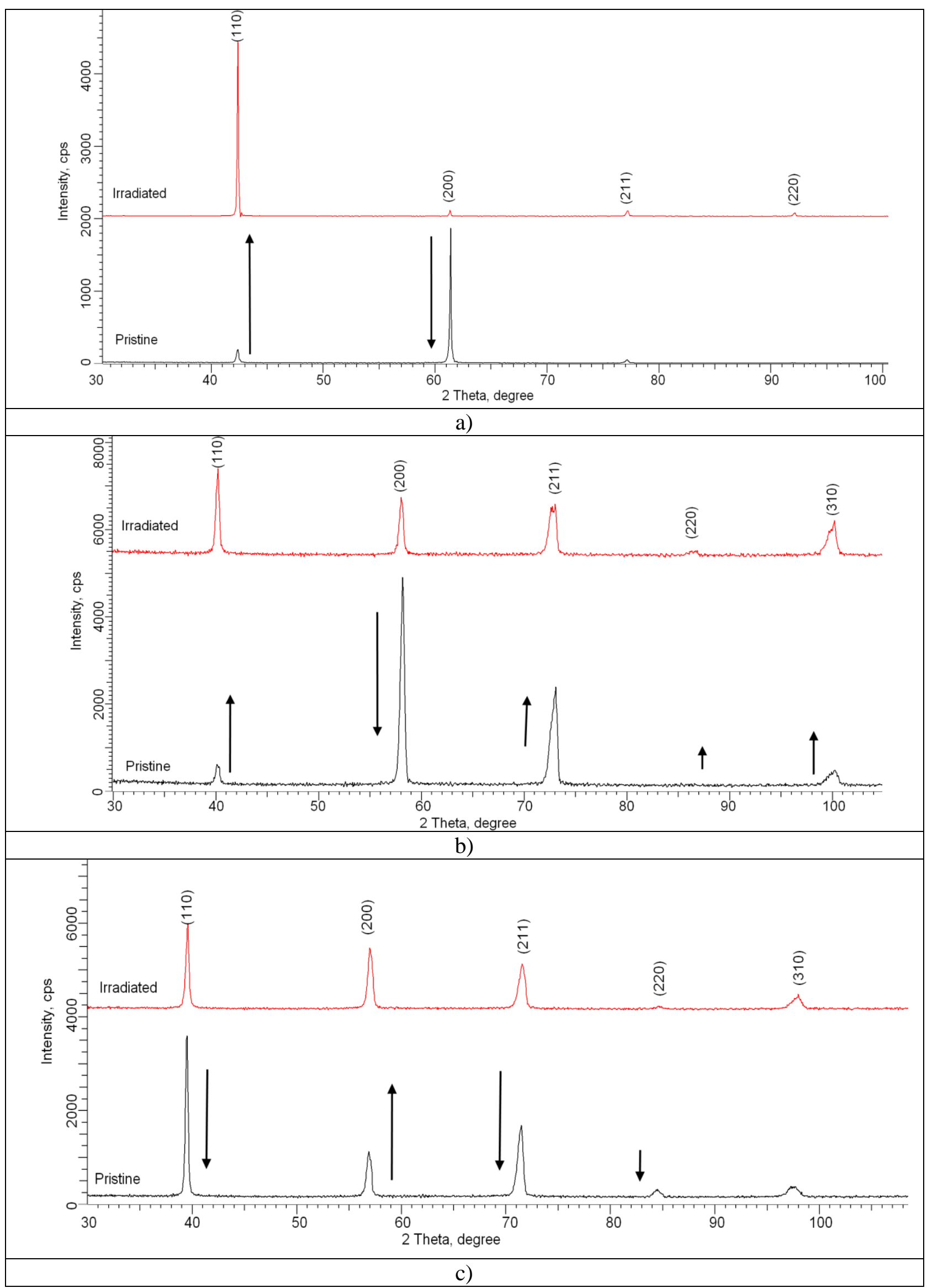




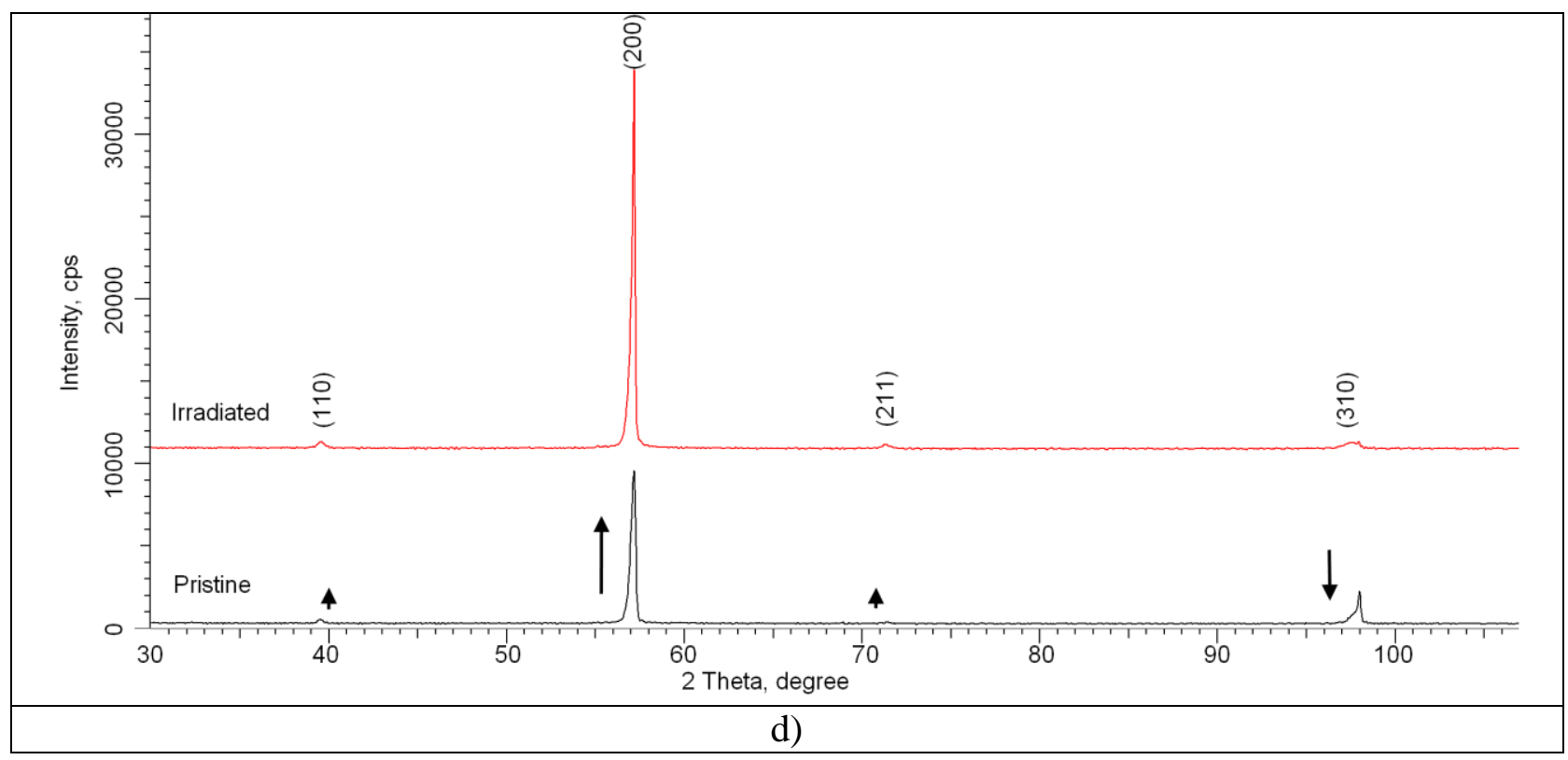

Figure 2. X-ray diffraction patterns of the samples under study before and after irradiation ("up" and "down" arrows denote the results of texture orientation changes that are estimated by reflex intensity changes): a) V alloys; b) NbV alloys; c) TaNbV alloys; d) TiTaNbV alloys

The addition of tantalum to the alloy leads to the formation of a substitutional solid solution structure of the TaNbV (PDF-01-071-9947) type with a body-centered crystal lattice and without a preferred orientation. For the irradiated TaNbV sample, in contrast to other samples, the preferred orientation does not change as a result of irradiation. At the same time, in the case of an irradiated sample, the width of the diffraction lines becomes slightly larger than for the sample in the initial state. It should also be noted that, in the case of this sample, the shift towards small angles of diffraction reflections is not as pronounced as in the case of samples $\mathrm{V}$ and $\mathrm{NbV}$ alloys. This behavior indicates an increase in the resistance to radiation swelling and deformation of the medium-entropy $\mathrm{TaNbV}$ alloy upon irradiation.

The addition of titanium to the TaNbV alloy leads to the formation of the TiTaNbV (PDF-01071-9947) substitutional solid solution phase, with the crystal lattice parameter $\mathrm{a}=3.2002 \AA$, while for the TaNbV alloy the crystal lattice parameter in the initial state was $a=3.2205 \AA$. This difference is primarily due to the difference in the atomic radii of titanium and tantalum. In this case, for the TiTaNbV alloy sample, as well as for TaNbV, in contrast to other samples, irradiation does not lead to the appearance of a reorientation effect, and the main changes in Xray diffraction patterns are associated only with a change in the intensity and width of diffraction lines before and after irradiation.

Figure 3 shows the assessment results of the change in the crystal lattice deformation and the swelling of the crystal lattice volume as a result of irradiation with $\mathrm{Kr}^{14+}$ ions. As can be seen from the data presented, pure vanadium has the highest degree of swelling and deformation of the crystal lattice. The addition of new components to the alloy composition and an increase in their number leads, as can be seen from the data in Figure 3, to an increase in stability, both to deformation and to swelling of the crystal lattice. This behavior of changes in structural parameters is primarily associated with the dislocation structure of the alloys, which significantly increases with an increase in the components in the alloy composition. Most likely, an increase in the components in high-entropy alloys leads to an increase in the dislocation density due to the formation of additional sinks of radiation defects, as well as to potential barriers leading to a change in the rate and energy of migration of radiation defects. At the same time, as can be seen from the data in Figure $3 \mathrm{~b}$, the increase in swelling resistance for $\mathrm{NbV}$ and $\mathrm{TaNbV}$ alloys in comparison with a pure alloy of pure vanadium is 2.5-4 times, while for a four-component alloy, the resistance value exceeds more than 20 times. A significant decrease in radiation swelling was 
also observed for medium- and high-entropy systems based on $\mathrm{Ni}(\mathrm{NiCoFe}$ and $\mathrm{NiCoFeCrMn})$ [19].

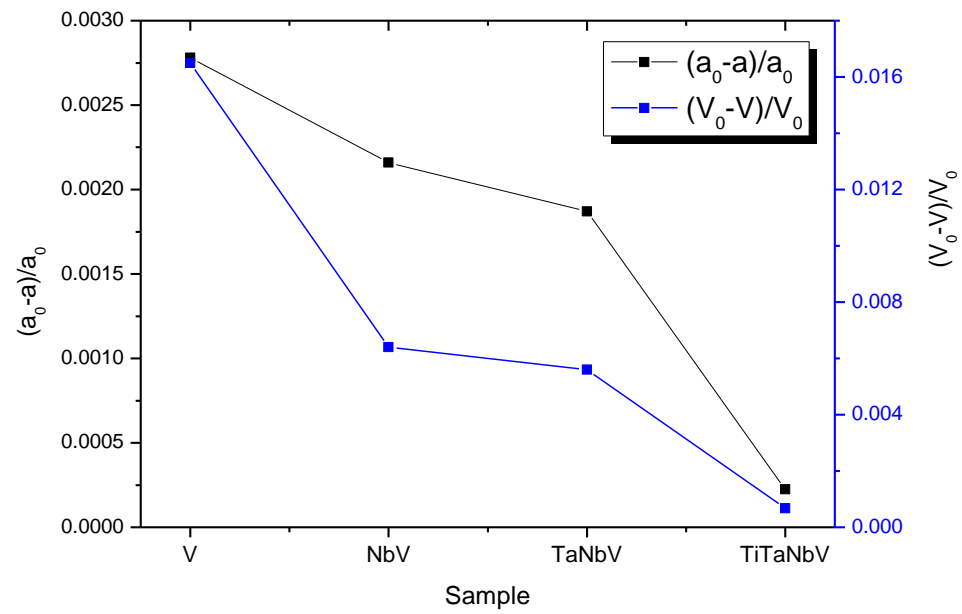

a)

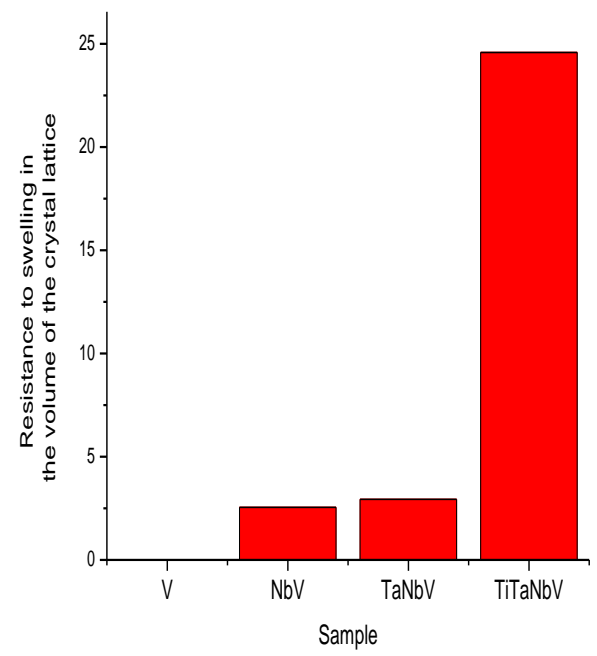

b)

Figure 3. a) Changes in deformation and swelling of the crystal lattice volume of $\mathrm{V}, \mathrm{NbV}$, TaNbV, TiTaNbV samples irradiated with $\mathrm{Kr}^{14+}$ ions with an energy of $280 \mathrm{keV}$; b) Diagram of the change in the swelling resistance value of the alloys crystal lattice volume with respect to $\mathrm{V}$

Figure 4 shows the assessment results of the deformation of interplanar distances for the two main (most intense) diffraction lines (110) and (200), the change in which reflects the reorientation of grains as a result of irradiation. As can be seen from the data presented, for all studied samples, the trends of changes for both diffraction lines are of the same nature, with a difference only in the magnitude of the deformation. Thus, the (110) line is characterized by deformation processes associated with the compression of interplanar distances, while the (200) line is characterized by tensile deformations. At the same time, an increase in the components in the composition of the alloys leads to a decrease in the deformation of the interplanar distances, and in the case of the TiTaNbV alloy, the deformation contributions are minimal, which indicates a high distortion resistance of the structure.

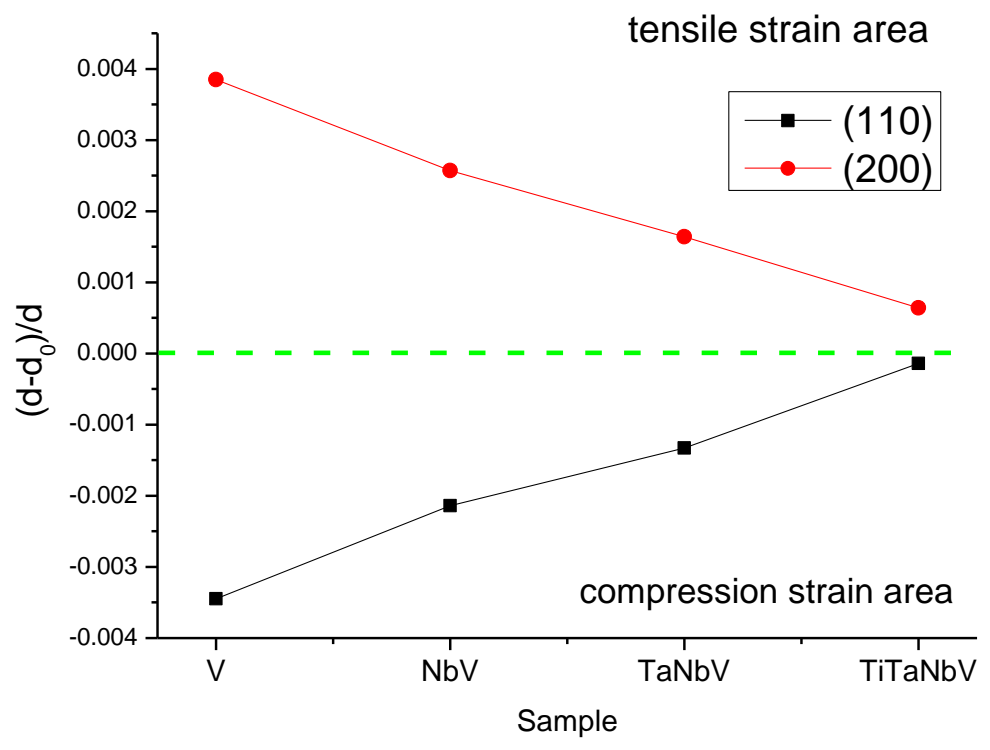

Figure 4. Changes in the interplanar distances deformation value for the (110) and (200) planes of the $\mathrm{V}, \mathrm{NbV}, \mathrm{TaNbV}$, TiTaNbV samples irradiated with krypton ions 
One of the important parameters affecting the applicability of alloys as structural materials are strength characteristics, in particular, the values of hardness and degree of softening depending on external influences. These characteristics make it possible to assess with high accuracy the resistance of materials to external influences, as well as the structural deformations and disordering caused by them. Figure 5 shows the results of changes in hardness before and after irradiation. As can be seen from the data presented, a change in the components in the composition of the initial alloys leads to an increase in hardness by a factor of 10-15 for compositions $\mathrm{NbV}$, TaNbV in comparison with a sample of pure vanadium. Such an increase in hardness for two and three-component alloys is in good agreement with the theory of highentropy alloys, according to which the addition of niobium or tantalum to the composition leads to an increase in the strength properties, as well as the density of the alloys. In turn, the addition of titanium to the alloy led to a decrease in the hardness values in the initial state. The decrease in alloy hardness in the case of titanium addition to the composition is due to the softening properties of titanium and lower hardness. Typically, titanium is added to increase the ductility of the alloys with small reductions in strength and hardness.

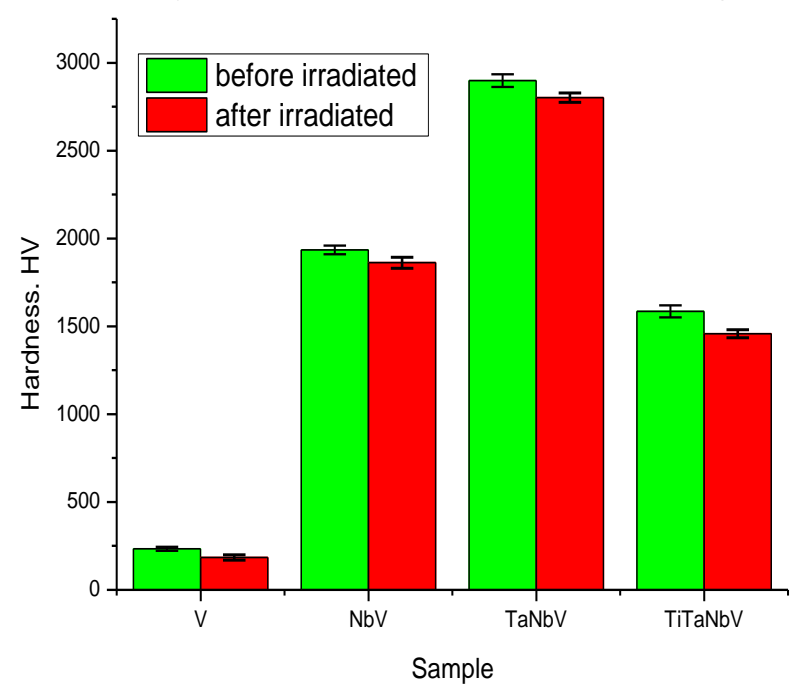

a)

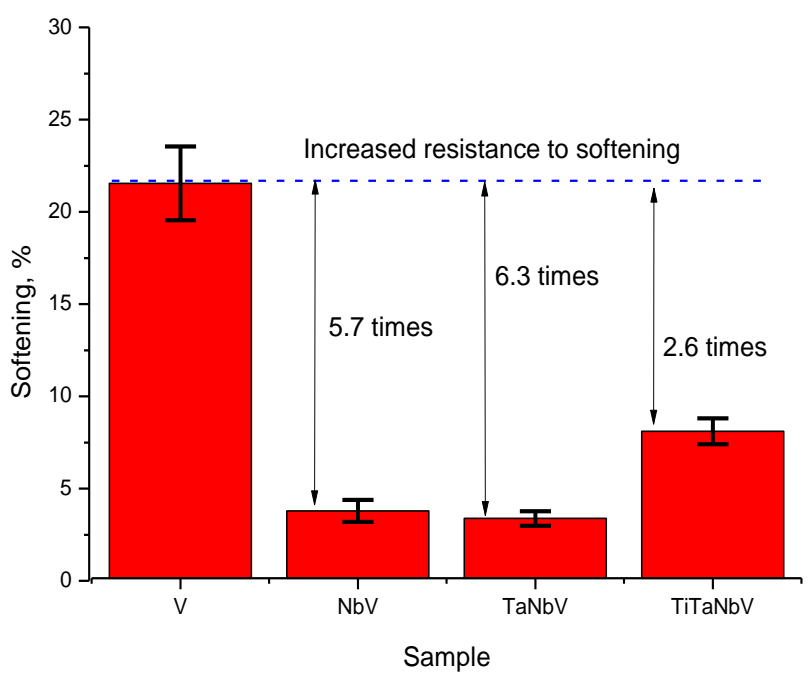

b)

Figure 5. a) Diagram of changes in hardness before and after irradiation; b) Dependence of the change in the swelling resistance degree and softening after irradiation with $280 \mathrm{keV} \mathrm{Kr}^{14+}$ ions

As can be seen from the data presented for irradiated samples, alloys based on $\mathrm{NbV}$ and $\mathrm{TaNbV}$ have the highest resistance to hardness reduction and softening, the stability of which is 5.7 and 6.7 times higher than that of pure vanadium alloy. At the same time, the TiTaNbV-based alloy has stability 2.6 times higher than the pure vanadium-based alloy. This behavior of decreasing hardness and softening resistance compared to $\mathrm{NbV}$ and $\mathrm{TaNbV}$ alloys is due to the softening properties of titanium when it is added to high-entropy alloys. It should be noted that the TiTaNbV alloy has higher resistance to deformation of the crystal lattice as a result of irradiation. The effect of irradiated samples softening is associated with the effect of radiation damage accumulation as a result of large displacements (30-50 dpa, depending on the type of alloy), as well as the implantation of low-energy $\mathrm{Kr}^{14+}$ ions into the structure (0.6-1.0 at.\%, according to calculations). At the same time, in the case of low-energy $\mathrm{Kr}^{14+}$ ions, the contribution from elastic and inelastic interactions of incident ions with the crystal lattice is equally probable, which causes a large value of atomic displacements.

\section{Conclusion}

As a result of the studies carried out, an increase in the resistance to swelling and deformation of the crystal structure in medium- and high-entropy $\mathrm{TaNbV}$ and TiTaNbV alloys was established. 
At the same time, alloys of the $\mathrm{NbV}$ and $\mathrm{TaNbV}$ type have the highest softening resistance degree, while the TiTaNbV alloy has a lower resistance to swelling, since the addition of titanium leads to a decrease in the density and hardness of the alloy.

Analyzing the data obtained, it can be concluded that the near-surface layer of medium- and high-entropy alloys $\mathrm{TaNbV}$ and $\mathrm{TiTaNbV}$ have a high degree of resistance to radiation damage under high-dose irradiation, as well as resistance to swelling and softening. Further studies will be aimed at studying the mechanisms of grain reorientation depending on the radiation dose.

\section{References}

1 George, Easo P., Dierk Raabe, and Robert O. Ritchie. "High-entropy alloys." Nature Reviews Materials 4.8 (2019): 515-534.

2 Miracle, Daniel B., and Oleg N. Senkov. "A critical review of high entropy alloys and related concepts." Acta Materialia 122 (2017): 448-511.

3 Zhang, Weiran, Peter K. Liaw, and Yong Zhang. "Science and technology in high-entropy alloys." Science China Materials 61.1 (2018): 2-22.

4 Wang, Xin, Wei Guo, and Yongzhu Fu. "High-entropy alloys: emerging materials for advanced functional applications." Journal of Materials Chemistry A 9.2 (2021): 663-701.

5 Kitagawa, Jiro, Shusuke Hamamoto, and Naoki Ishizu. "Cutting edge of high-entropy alloy superconductors from the perspective of materials research." Metals 10.8 (2020): 1078.

6 Troparevsky, M. Claudia, et al. "Criteria for predicting the formation of single-phase highentropy alloys." Physical Review X 5.1 (2015): 011041.

7 Steurer, Walter. "Single-phase high-entropy alloys-A critical update." Materials Characterization 162 (2020): 110179.

8 Tian, Fuyang, et al. "Empirical design of single phase high-entropy alloys with high hardness." Intermetallics 58 (2015): 1-6.

9 Zhou, Naixie, et al. "Single-phase high-entropy intermetallic compounds (HEICs): bridging high-entropy alloys and ceramics."Science Bulletin 64.12 (2019): 856-864.

10 Otto, Frederik, et al. "Decomposition of the single-phase high-entropy alloy CrMnFeCoNi after prolonged anneals at intermediate temperatures." Acta Materialia 112 (2016): 40-52.

11 Xia, S. Q., et al. "Irradiation resistance in $\mathrm{Al}$ x CoCrFeNi high entropy alloys." Jom 67.10 (2015): 2340-2344.

12 Xia, Song-qin, et al. "Irradiation behavior in high entropy alloys."Journal of Iron and Steel Research, International 22.10 (2015): 879-884.

13 Kadyrzhanov, K. K., K. Tinishbaeva, and V. V. Uglov. "Investigation of the effect of exposure to heavy Xe22+ ions on the mechanical properties of carbide ceramics." Eurasian Phys. Tech. J 17 (2020): 46-53.

14 Egami, Takeshi, et al. "Local electronic effects and irradiation resistance in high-entropy alloys." Jom 67.10 (2015): 2345-2349.

15 Chen, Wei-Ying, et al. "Irradiation effects in high entropy alloys and $316 \mathrm{H}$ stainless steel at $300^{\circ}$ C." Journal of Nuclear Materials 510 (2018): 421-430.

16 Yang, Tengfei, et al. "Effects of temperature on the irradiation responses of $\mathrm{Al} 0.1 \mathrm{CoCrFeNi}$ high entropy alloy." Scripta Materialia144 (2018): 31-35.

17 Kumar, NAP Kiran, et al. "Microstructural stability and mechanical behavior of FeNiMnCr high entropy alloy under ion irradiation." Acta Materialia 113 (2016): 230-244.

18 Yang, Tengfei, et al. "Precipitation behavior of $\mathrm{Al}$ x CoCrFeNi high entropy alloys under ion irradiation." Scientific reports 6.1 (2016): 1-8.

19 Jin, K., et al. "Effects of compositional complexity on the ion-irradiation induced swelling and hardening in Ni-containing equiatomic alloys." Scripta Materialia 119 (2016): 65-70.

20 Nagase, Takeshi, et al. "Electron-irradiation-induced structural change in $\mathrm{Zr}-\mathrm{Hf}-\mathrm{Nb}$ alloy." Intermetallics 26 (2012): 122-130. 
21 Liang, Hui, et al. "Microstructure and tribological properties of AlCrFe2Ni2W0. 2Mo0. 75 high-entropy alloy coating prepared by laser cladding in seawater, $\mathrm{NaCl}$ solution and deionized water."Surface and Coatings Technology 400 (2020): 126214.

$22 \mathrm{Gu}$, Zhen, et al. "Microstructure and wear behavior of mechanically alloyed powder AlxMo0. $5 \mathrm{NbFeTiMn} 2$ high entropy alloy coating formed by laser cladding." Surface and Coatings Technology 401 (2020): 126244.

$23 \mathrm{Liu}, \mathrm{Hao}$, et al. "Effect of heat treatment on phase stability and wear behavior of laser clad AlCoCrFeNiTi0. 8 high-entropy alloy coatings." Surface and Coatings Technology 392 (2020): 125758.

$24 \mathrm{Lu}$, Jie, et al. "Y-Hf co-doped AlCoCrFeNi high-entropy alloy coating with superior oxidation and spallation resistance at $1100^{\circ}$ C." Corrosion Science 182 (2021): 109267.

25 Meghwal, Ashok, et al. "Thermal spray high-entropy alloy coatings: a review." Journal of Thermal Spray Technology 29 (2020): 857-893.

26 Ziegler, James F., Matthias D. Ziegler, and Jochen P. Biersack. "SRIM-The stopping and range of ions in matter (2010)." Nuclear Instruments and Methods in Physics Research Section B: Beam Interactions with Materials and Atoms 268.11-12 (2010): 1818-1823. 